TSUKUBA J. MATH.

Vol. 8 No. 1 (1984). $69-75$

\title{
ON CONDUCTOR OVERRINGS OF AN INTEGRAL DOMAIN
}

by

\section{Akira OKABE}

InTRODUCTION. Throughout this paper, $R$ will be an integral domain with identity, and $K$ will be the quotient field of $R$. By an overring of $R$ we shall mean any integral domain $S$ between $R$ and $K$. A proper overring of $R$ is an overring $S$ such that $R \neq S$. Any unexplained terminology is standard as in [3] or [6].

If $I$ is an ideal of $R$, then $I:{ }_{K} I=\{x \in K \mid x I \subseteq I\}$ is an overring of $R$ and furthermore it is a subring of the ideal transform $T(I)=\bigcup_{n=1}^{\infty}\left\{x \in K \mid x I^{n} \subseteq R\right\}$. We shall call $I:{ }_{K} I$ the conductor overring of $R$ with respect to $I$.

In [8] Nagata has shown that if $I$ is an ideal of $R$ and $R^{\prime}$ is an overring of $R$ such that $R \subseteq R^{\prime} \subseteq T(I)$, then there exists a one-one correspondence between the set of all prime ideals $P^{\prime}$ of $R^{\prime}$ not containing $I R^{\prime}$ and the set of all prime ideals $P$ of $R$ not containing $I$. Furthermore, this correspondence can be realized in such a manner that if $P$ corresponds to $P^{\prime}$, then $P=P^{\prime} \cap R$ and $R_{P}=R^{\prime}{ }_{P \prime}$. Hence, if $I$ is an ideal of $R$ then $P^{\prime} \rightarrow P^{\prime} \cap R$ is a one-one mapping from the set of all prime ideals $P^{\prime}$ of $I:{ }_{K} I$ not containing $I$ onto the set of all prime ideals $P$ of $R$ not containing $I$.

Our results are divided into two sections. In Section 1 we show that if $I$ is an ideal of $R$ then $P \rightarrow(P \cap I):{ }_{K} I$ gives a one-one correspondence between the set of all prime ideals $P$ of $R$ not containing $I$ and the set of all prime ideals $P^{\prime}$ of $I:{ }_{K} I$ not containing $I$.

In Section 2 we prove that if $I$ is an ideal of $R$ and $P$ is a prime ideal of $R$ not containing $I$, then $I:{ }_{K} I /\left((P \cap I):{ }_{K} I\right)$ is isomorphic to a subring of $(I+P) / P$ : ${ }_{L}(I+P) / P$ with $L$ the quotient field of $R / P$. As a corollary, it will be shown that if $P$ is a prime ideal of $R$ properly contained in an ideal $I$ of $R$, then $P:{ }_{K} I$ is not a maximal ideal of $I:{ }_{K} I$.

\section{SOME PRELIMINARY RESULTS}

We first establish some general results concerning conductor overrings.

LEMMA 1.1. Let $I$ be an ideal of $R$ and let $S$ be a proper overring of $R$. (1) $I$ is an ideal of $I:{ }_{K} I$. 
(2) If we set $I_{(S)}=R:{ }_{R} S$, then $S \subseteq I_{(S)}:{ }_{K} I_{(S)}$.

(3) If $J$ is an ideal of $R$ such that $S \subseteq J:_{K} J$, then $J \subseteq I_{(S)}$.

(4) If $I$ is also an ideal of $S$, then $S \subseteq I:{ }_{K} I$ and $I \subseteq I_{(S)}$.

(5) $I:{ }_{R} S$ is an ideal of $R$ and is contained in $I$. Furthermore if $I$ is also an ideal of $S$, then $I=I:{ }_{R} S$.

(6) $I=I:{ }_{R}\left(I:{ }_{K} I\right)$.

(7) If $J$ is an ideal of $R$ such that $J \subset I$, then $J:{ }_{K} I$ is a proper ideal of $I:{ }_{K} I$.

Proof. (1) This is trivial.

(2) We first show that $I_{(S)}$ is an ideal of $S$. Let $x, y \in I_{(S)}$ and $s \in S$. Since $x S \subseteq R$ and $y S \subseteq R,(x-y) S \subseteq x S+y S \subseteq R$, and so $x-y \in I_{(S)}$. Next, since $x \in I_{(S)}$ and $s \in S, x s \in R$ and moreover $s S \subseteq S$, and therefore $(x s) S=x(s S) \subseteq x S \subseteq R$. Thus $x s \in I_{(S)}$, and therefore $I_{(S)}$ is an ideal of $S$ as we required. Then, since $I_{(S)}$ is an ideal of $S$, it is clear that $S \subseteq I_{(S)}:{ }_{K} I_{(S)}$.

(3) By hypothesis, $J S \subseteq J \subseteq R$ and so $J \subseteq I_{(S)}$.

(4) The first assertion is evident. Next, since $S \subseteq I:{ }_{K} I$, the second assertion follows immediately from (3).

(5) Let $x, y \in I:{ }_{R} S$ and $r \in R$. Then $x S \subseteq I$ and $y S \subseteq I$, and so $(x-y) S \subseteq x S+$ $y S \subseteq I$. Thus $x-y \in I:{ }_{R} S$. Next, since $(r x) S=r(x S) \subseteq r I \subseteq I, r x \in I:{ }_{R} S$. Thus $I:{ }_{R} S$ is an ideal of $R$. Moreover, if $x \in I:{ }_{R} S$ then $x=x 1 \in x S \subseteq I$, and hence $I:{ }_{R} S \subseteq I$. Assume furthermore that $I$ is an ideal of $S$. If $x \in I$, then $x S \subseteq I$ and so $I \subseteq I:{ }_{R} S$. Hence we have $I=I:{ }_{R} S$ as we wanted.

(6) Since $I$ is an ideal of $I:{ }_{K} I$, our assertion follows from (5).

(7) Let $x, y \in J:{ }_{K} I$ and $t \in I:{ }_{K} I$. Then $x I \subseteq J$ and $y I \subseteq J$, and therefore $(x-y) I$ $\subseteq x I+y I \subseteq J$. Thus $x-y \in J:{ }_{K} I$. Next, $(x t) I=x(t I) \subseteq x I \subseteq J$ and hence $x t \in J:_{K} I$. Therefore $J:_{K} I$ is an ideal of $I{ }_{K} I$. Assume that $J:_{K} I=I:{ }_{K} I$. Then, since $1 \in I:{ }_{K} I=J:{ }_{K} I, I=1 I \subseteq J$ and so $I=J$, a contradiction. Therefore $J:{ }_{K} I$ is a proper ideal of $I:{ }_{K} I$.

REMARK 1.2. Let $I$ be a proper ideal of $R$. Then $I:{ }_{K} I=K$ if and only if $I=(0)$. If $I:{ }_{K} I=K$, then, by (1) of Lemma $1.1, I$ is an ideal of a field $K$ and hence $I=(0)$. Conversely, if $I=(0)$, then clearly $I:{ }_{K} I=(0):{ }_{K}(0)=K$.

Proposition 1.3. Let $I$ be a nonzero ideal of $R$ and let $P$ be a prime ideal of $R$ not containing $I$. Then

(1) $(P \cap I):{ }_{K} I=\{x \in K \mid x I \subseteq P \cap I\}$ is a prime ideal of $I:{ }_{K} I$.

(2) $\cdot\left((P \cap I):{ }_{K} I\right) \cap R=P$.

(3) If $P^{\prime}$ is a prime ideal of $I:{ }_{K} I$ such that $P^{\prime} \cap R=P$, then $P^{\prime}=(P \cap I):{ }_{K} I$.

(4) $R_{P}=\left(I:{ }_{K} I\right)_{\left((P \cap I): K_{K} I\right)}$. 
Proof. (1) By Lemma $1.1(7),(P \cap I):{ }_{K} I$ is a proper ideal of $I:{ }_{K} I$. We shall next prove that $(P \cap I):{ }_{K} I$ is a prime ideal of $I:_{K} I$. To prove this, let $x, y \in I:{ }_{K} I$, $x y \in(P \cap I):{ }_{K} I$, and $x \notin(P \cap I):{ }_{K} I$. First, since $x \notin(P \cap I):{ }_{K} I$, there exists an element $t \in I$ such that $x t \notin P \cap I$. Then $x t \notin P$, because $x t \in\left(I:{ }_{K} I\right) I \subseteq I$. Now, if $r \in I$, then $r t \in I$, and hence $(x t)(y r)=(x y)(t r) \in P \cap I \subseteq P$. But then, since $x t \in R \backslash P$, yre $\left(I:{ }_{K} I\right) I \subseteq I \subset R,(x t)(y r) \in P$ implies that $y r \in P$. Thus $y I \subseteq P$ and therefore $y I \subseteq P \cap I$, that is, $y \in(P \cap I):{ }_{K} I$. Hence it follows that $(P \cap I):_{K} I$ is a prime ideal of $I:{ }_{K} I$.

(2) The containment $P \subseteq\left((P \cap I):{ }_{K} I\right) \cap R$ is clear. To prove the reverse containment, let $x \in\left((P \cap I):{ }_{K} I\right) \cap R$. Choose $t \in I \backslash P$. Then $t \in R \backslash P, x \in R$, and $x t \in P$ and so $x \in P$, because $P$ is a prime ideal of $R$.

(3) and (4) follow from Nagata's theorem mentioned in Introduction, but here we give direct proves.

(3) First, let $x \in P^{\prime}$. Since $P^{\prime}$ is an ideal of $I:{ }_{K} I, x I \subseteq I \cap P^{\prime}=I \cap\left(R \cap P^{\prime}\right)=I \cap P$ and so $x \in(P \cap I):{ }_{k} I$. Thus $P^{\prime} \subseteq(P \cap I):{ }_{k} I$. Conversely, let $x \in(P \cap I):{ }_{k} I$. Then $x I \subseteq P \cap I=P^{\prime} \cap I$. Choose $t \in I \backslash P$. Then $x t \in P^{\prime} \cap I \subseteq P^{\prime}, t \notin P^{\prime}$, and $x \in I:{ }_{K} I$ and so $x \in P^{\prime}$, because $P^{\prime}$ is a prime ideal of $I:{ }_{K} I$. Thus we also have $(P \cap I):{ }_{K} I \subseteq P^{\prime}$.

To prove (4), we need the following

LEMMA 1.4. If $I$ is an ideal of $R$ and $P$ is a prime ideal of $R$ not containing $I$, then $P \cap I$ is also an ideal of $I:{ }_{K} I$.

Proof. To prove this, we need to show that $(P \cap I)\left(I:{ }_{K} I\right)=P \cap I$. The containment $P \cap I \subseteq(P \cap I)\left(I{ }_{k} I\right)$ is clear. To prove the reverse containment, let $x \in(P \cap I)\left(I:{ }_{K} I\right)$. Then we can write $x=\sum a_{i} x_{i}$, where $a_{i} \in P \cap I$ and $x_{i} \in I:{ }_{K} I$. Now if we choose $s \in I \backslash P$, then $x_{i} s \in\left(I:{ }_{K} I\right) I \subseteq I \subseteq R$. Hence $x s=\sum a_{i}\left(x_{i} s\right) \in P \cap I \subseteq P$. But, since $x \in(P \cap I)\left(I:{ }_{K} I\right) \subseteq I \subseteq R$ and $s \in R \backslash P, x s \in P$ implies that $x \in P$. Thus $x \in P \cap I$, as desired.

Now let us return to the proof of (4) in Proposition 1.3. Since $P=((P \cap I)$ : $\left.{ }_{K} I\right) \cap R, R_{P} \subseteq\left(I:{ }_{K} I\right)_{((P \cap I):}{ }_{K}^{I)}$. Conversely, let $\left.x \in\left(I:_{K} I\right)_{((P \cap I):}{ }_{K} I\right)$. Then we can write $x=a / b$, where $a \in I:{ }_{K} I$ and $b \notin(P \cap I):{ }_{K} I$. Since $b \notin(P \cap I):{ }_{K} I$, there exists $t \in I$ such that $b t \notin P \cap I$. Then necessarily $t \notin P$. Assume the contrary. Then, since $t \in I \cap P, b t \in(P \cap I)\left(I:{ }_{K} I\right)=P \cap I$, a contradiction. Thus $t \notin P$ and therefore $t \notin(P \cap I):{ }_{K} I$. Then $x=a t / b t \in R_{P}$, because $a t \in I \subseteq R$ and $b t \in I \backslash P \subseteq R \backslash P$. This completes our proof.

Corollary 1.5. Let I be an ideal of $R$ and let $P$ be a prime ideal of $R$ not containing $I$. Then $P$ is a prime ideal of $I:{ }_{K} I$ if and only if $P=(P \cap I):{ }_{K} I$. In particular, if $P$ is properly contained in $I$, then $P$ is a prime ideal of $I:{ }_{K} I$ if and only if $P=P:{ }_{K} I$. 
Proof. This follows immediately from Proposition 1.3.

REMARK 1.6. If $J \subset I$ are ideals of $R$, then $J$ is not necessarily an ideal of $I:{ }_{K} 1$. For example, let $k$ be a field and $R=k\left[X^{2}, X^{3}\right]$ be the subring of $k[X]$. Then $K=k(X)$ is the quotient field of $R$. If we set $M=X^{2} R+X^{3} R$, then $M$ is a maximal ideal of $R$ and $M:{ }_{K} M=k[X] \neq R$. Furthermore, if we take $I=X^{3} R \subset M$, then $I$ is not an ideal of $M:{ }_{K} M$, because $X^{3} \in I, X \in M:{ }_{K} M$, but $X^{3} X=X^{4} \notin I$.

\section{THE MAIN THEOREM}

Lemma 2.1. Let $I$ be an ideal of $R$ and let $P$ be a prime ideal of $R$ not containing $I$. Then, for an element $x \in I:{ }_{K} I, x \notin(P \cap I):{ }_{K} I$ if and only if $x t \notin P \cap I$ for all $t \in I \backslash P$.

Proof. The "if" half is trivial. Conversely, suppose that $x \notin(P \cap I):{ }_{K} I$. Then there exists $t_{0} \in I$ such that $x t_{0} \notin P \cap I$. Since $x t_{0} \in\left(I:_{K} I\right) I \subseteq I, x t_{0} \notin P$. Moreover, it follows that $t_{0} \notin P$. Suppose the contrary. Then, $x t_{0} \in(P \cap I)\left(I:{ }_{K} I\right)=P \cap I$ by Lemma 1.4. But this contradicts the choice of $t_{0}$. Thus $t_{0} \notin P$ as required. Then, for any element $t \in I \backslash P, x t \in I \subseteq R$ and $(x t) t_{0}=\left(x t_{0}\right) t \notin P$, and therefore $x t \notin P$. Thus $x t \notin P \cap I$ for all $t \in I \backslash P$, and the proof is completed.

Now we shall prove the main theorem.

THEOREM 2.2. Let $I$ be an ideal of $R$ and let $P$ be a prime ideal of $R$ not containing $I$. Then $I:{ }_{K} I /\left((P \cap I):{ }_{K} I\right)$ is isomorphic to a subring of $(I+P) / P$ : ${ }_{L}(I+P) / P$, where $L$ is the quotient field of $R / P$.

Proof. For each $x \in I:{ }_{K} I$, we shall denote its coset $x+(P \cap I):{ }_{K} I$ by $\tilde{x}$, and, for each $r \in R$, we shall denote its coset $r+P$ by $\bar{r}$. Now we shall first define a mapping $\Phi$ of $I:{ }_{K} I /\left((P \cap I):{ }_{K} I\right)$ into $(I+P) / P:_{L}(I+P) / P$ as follows: For each $\tilde{x} \in I:{ }_{K} I /\left((P \cap I):{ }_{K} I\right)$, we set $\Phi(\tilde{x})=\overline{x t} / \bar{t}$ where $t \in I \backslash P$. Let us first show that this mapping $\Phi$ is well-defined. If $t$ and $u$ are any two elements of $I \backslash P$, then $\bar{t} \overline{x u}=$ $\overline{t x u}=\overline{x t} \bar{u}$ in $R / P$, and so $\overline{x t} / \bar{t}=\overline{x u} / \bar{u}$ in $L$. Next, if $y$ is any other representative of the coset $\tilde{x}$, then $x-y \in(P \cap I):{ }_{K} I$, and so, for any $t \in I \backslash P,(x-y) t \in P \cap I \subseteq P$. Hence $\overline{x t}=\overline{y t}$ in $R / P$, and so, $\overline{x t} / \bar{t}=\overline{y t} / \bar{t}$ in $L$. Thus $\Phi(\tilde{x})=\overline{x t} / \bar{t}$ does depend only on the coset $\tilde{x}$, and not on the choice of a representative of $\tilde{x}$ and an element $t$ of $I \backslash P$, and therefore the mapping $\Phi$ is well-defined. Next, let us show that $\Phi$ is a ring homomorphism from $I:{ }_{K} I /\left((P \cap I):{ }_{K} I\right)$ into $L$. To prove this, let $\tilde{x}, \tilde{y} \in I$ : 
${ }_{K} I /\left((P \cap I):{ }_{K} I\right)$. Then, for any $t \in I \backslash P,(\overline{x+y) t} / \bar{t}=(\overline{x t+y t}) / \bar{t}=(\overline{x t}+\overline{y t}) / \bar{t}=\overline{x t} / \bar{t}+\overline{y t} / \bar{t}$, and hence it follows that $\Phi(\tilde{x}+\tilde{y})=\Phi(\widetilde{x+y})=\overline{(x+y) t} / \bar{t}=\overline{x t} / \bar{t}+\overline{y t} / \bar{t}=\Phi(\tilde{x})+\Phi(\tilde{y})$. Moreover, for any $t \in I \backslash P, \overline{x y t^{2}} / \overline{t^{2}}=\overline{(x t)(y t)} / \bar{t}^{2}=(\overline{x t} / \bar{t})(\overline{y t} / \bar{t})$, and so we have $\Phi(\tilde{x} \tilde{y})=\Phi(\tilde{x y})$ $=\overline{x y t^{2}} / \overline{t^{2}}=(\overline{x t} / \bar{t})(\overline{y t} / \bar{t})=\Phi(\tilde{x}) \cdot \Phi(\tilde{y})$. Thus $\Phi$ is a ring homomorphism from $I:_{K} I /$ $\left((P \cap I):{ }_{K} I\right)$ into $L$. We shall now proceed to prove the injectivity of $\Phi$. For this, assume that $\Phi(\tilde{x})=0$ for some coset $\tilde{x}$. Then $\bar{x} t=0$ in $R / P$ for all $t \in I \backslash P$, so that $x t \in P \cap I$ for all $t \in I \backslash P$. Moreover, for any $s \in P \cap I$, we have, by Lemma 1.4, $x s \in(P \cap I)\left(I:{ }_{K} I\right)=P \cap I$. Hence it follows that $x I \subseteq P \cap I$, that is, $x \in(P \cap I):_{K} I$, and so $\tilde{x}=0$ in $I:{ }_{K} I /\left((P \cap I):{ }_{K} I\right)$ as we asserted. It now remains to show that the image of $I:{ }_{K} I /\left((P \cap I):{ }_{K} I\right)$ under $\Phi$ is actually contained in $(I+P) / P:{ }_{L}(I+P) / P$. To prove this, let $\tilde{x} \in I:{ }_{k} I /\left((P \cap I):{ }_{K} I\right)$. Then, for any $\bar{r} \in(I+P) / P$ with $r \in I$, we have $\Phi(\tilde{x}) \bar{r}=((\overline{x t}) / \bar{t}) \bar{r}=\overline{x t r} / \bar{t}=\overline{x r} \cdot \bar{t} / \bar{t}=\overline{x r} \in(I+P) / P$, where $t \in I \backslash P$. Thus we have $\Phi(\tilde{x}) \epsilon(I+P) / P:{ }_{L}(I+P) / P$ for all $\tilde{x} \in I:{ }_{K} I /\left((P \cap I):{ }_{k} I\right.$, and accordingly, $\operatorname{Im}(\Phi)$ is actually contained in $(I+P) / P:_{L}(I+P) / P$. Thus our proof is complete.

REMARK 2.3. It would be worth noting that the ring homomorphism $\Phi$ defined in Theorem 2.2 is the identity mapping on $R / P$. In fact, by Proposition 1.3, $R / P \subseteq I:{ }_{K} I /\left((P \cap I):{ }_{K} I\right)$ and moreover, for any $\bar{r} \in R / P, \Phi(\bar{r})=\overline{r t} / \bar{t}=\bar{r} \cdot \bar{t} / \bar{t}=\bar{r}$ where $t \in I \backslash P$. Thus $\Phi$ is the identity mapping on $R / P$.

Corollary 2.4. Let $I$ be an ideal of $R$ and let $P$ be a prime ideal of $R$ not containing $I$. Then

(1) If $I+P=R$, then $I:{ }_{K} I /\left((P \cap I):_{K} I\right.$ is isomorphic to $R / P$.

(2) $P$ is a maximal ideal of $R$ if and only if $(P \cap I):{ }_{K} I$ is a maximal ideal of $I:{ }_{K} I$.

Proof. (1) If $I+P=R$, then we have $(I+P) / P:{ }_{L}(I+P) P=R / P:{ }_{L} R / P=R / P$, where $L$ is the quotient field of $R / P$, and then by Theorem 2.2 and Remark 2.3, $I:{ }_{K} I /\left((P \cap I):{ }_{K} I\right)$ is isomorphic to $R / P$ as we asserted.

(2) If $P$ is a maximal ideal of $R$, then $I+P=R$, since $I$ is not contained in $P$. Then, by the above result (1), $I{ }_{K} I /\left((P \cap I):{ }_{K} I\right)$ is a field, and accordingly, $(P \cap I):{ }_{K} I$ is a maximal ideal of $I:{ }_{k} I$. Conversely, assume that $(P \cap I):{ }_{k} I$ is a maximal ideal of $I:{ }_{K} I$. If $I+P=R$, then, by the above result (1), $P$ is also a maximal ideal of $R$. Hence, to prove that $P$ is a maximal ideal of $R$, it suffices to show that $I+P=R$. We shall now recall that by Theorem 2.2 and Remark 2.3, $I:{ }_{K} I /\left((P \cap I):{ }_{K} I\right)$ is isomorphic to an integral domain $T$ which is an overring of $R / P$ and is contained in $(I+P) / P:{ }_{L}(I+P) / P$. Now, by our assumption, $T$ is a field, and so $T=(I+P) / P:{ }_{L}(I+P) / P=L$. Hence, if $I+P$ is a proper ideal of $R$, 
then, by Remark 1.2, $(I+P) / P=(0)$ in $R / P$ and therefore $I \subseteq P$, a contradiction. Therefore we have $I+P=R$, as we wanted.

Corollary 2.5. If $P$ is a prime ideal of $R$, then $\operatorname{dim} P:{ }_{K} P \geqq \operatorname{rank} P$.

Proof. If $P=(0)$, then, by Remark $1.2,(0):{ }_{K}(0)=K$ and hence $\operatorname{dim}(0):{ }_{K}(0)=$ $\operatorname{rank}(0)=0$, whence our corollary is valid. Then assume that $P$ is a nonzero prime ideal of rank $r<\infty$, and let $(0) \subset P_{1} \subset P_{2} \subset \cdots \subset P_{r-1} \subset P$ be a chain of distinct prime ideals of $R$. By Proposition 1.3, (0)=(0): ${ }_{K} P \subset P_{1}:{ }_{K} P \subset \cdots \subset P_{r-1}:_{K} P$ is a chain of distinct proper prime ideals of $P:{ }_{K} P$. Since, by Corollary $2.4, P_{r-1}:_{K} P$ is not a maximal ideal of $P:{ }_{K} P$, the ideal $P_{r-1}:_{K} P$ is properly contained in a maximal ideal $M$ of $P:{ }_{k} P$. Then

$$
(0) \subset P_{1}:{ }_{K} P \subset P_{2}:{ }_{K} P \subset \cdots \subset P_{r-1}:{ }_{K} P \subset M
$$

is a chain of length $r$ of distinct proper prime ideals of $P:{ }_{K} P$. The assertion follows immediately from this fact. Lastly, we assume that rank $P$ is infinite. Then, as in the case of finite rank, it follows from Proposition 1.3 and Corollary 2.4 that $\operatorname{dim} P:{ }_{K} P$ is infinite, and hence our proof is complete.

REMARK 2.6. If $P$ is a finitely generated prime ideal of $R$, then Corollary 2.5 is evident. For, in this case, $P:{ }_{k} P$ is integral over $R$, and accordingly dim $P:{ }_{K} P=\operatorname{dim} R \geqq \operatorname{rank} P$.

Corollary 2.7. Let $(R, M)$ be a quasi-local domain. Then every maximal ideal of $M:{ }_{K} M$ lies over $M$.

Proof. Let $P$ be an arbitrary maximal ideal of $M:{ }_{K} M$. Then we always have $P \cap R \subseteq M$. If $Q=P \cap R \neq M$, then, by Proposition 1.3, $P=Q:{ }_{K} M$. But then, by Corollary $2.4, P$ is not a maximal ideal of $M:{ }_{K} M$, the desired contradiction. Thus we have $P \cap R=M$, as asserted.

\section{References}

[1] Arnold, J. T. and Brewer, J. W., On flat overrings, ideal transforms and generalized transforms of a commutative ring, J. Algebra, 18 (1971), 254-263.

[2] Fossum, R.M., The Divisor Class Group of a Krull Domain, Springer-Verlag, New York, 1973.

[3] Gilmer, R., Multiplicative Ideal Theory, Marcel Dekker, Inc., New York, 1972.

[4] - Multiplicative Ideal Theory, Queen's Papers in Pure and Applied Mathematics, No. 12, Queen's Univ. Press, Kingston, Ontario, 1968.

[5] Huckaba, J.A. and Papick, I. J., When the dual of an ideal is a ring, Manuscripta Math., 37 (1982), 67-85.

[6] Kaplansky, I., Commutative Rings, Allyn and Bacon, Inc., Boston, 1970. 
[ 7 J Larsen, M. D. and McCarthy, P. J., Multiplicative theory of ideals, Academic Press, New York and London, 1971.

| $8 \mid$ Nagata, M., A treatise on the 14-th problem of Hilbert, Mem. Coll. Sci. Univ. Kyoto Ser. A Math., 30 (1956-57), 57-70.

Gunma Technical College

Toba-cho, Maebashi

371 Japan 